\title{
LA EVALUACIÓN DEL DESEMPEÑO FERIAL DE LOS EXPOSITORES EN FERIAS INTERNACIONALES EN EL PERÚ: EL CASO \\ DE LOS EXPOSITORES EN LAS FERIAS INTERNACIONALES PERÚ MODA Y LA PERÚ GIFT DEL AÑO 2010
}

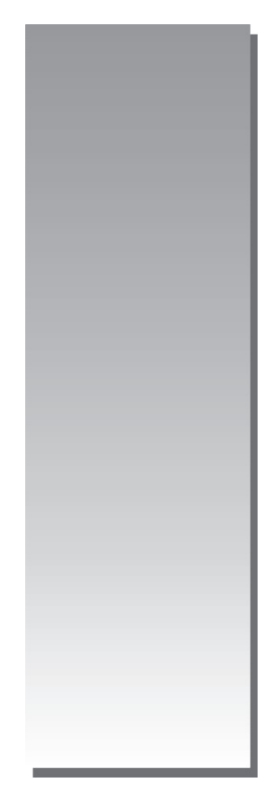

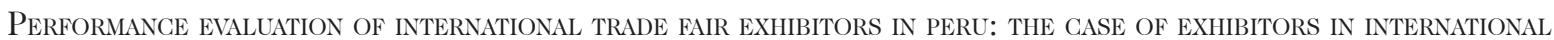
TRADE FAIRS PERU MODA AND PERU GIFT OF THE YEAR 2010

Enrique Angles Sánchez* enrique.angles@tu-berlin.de

[RECEPCIÓN: ABRIL 2016 / CONFORMIDAD: MAYO 2016]

\section{RESUMEN}

Las ferias internacionales son un instrumento de marketing internacional generalmente usadas por las empresas exportadoras. A pesar de las ventajas que presentan las ferias internacionales, las empresas exportadoras peruanas tienen una limitada participación en ellas. No existe evidencia que muestre cómo las empresas expositoras peruanas evalúan su participación en ferias internacionales. El objetivo principal del presente estudio es analizar si las empresas expositoras evalúan su desempeño ferial en las ferias internacionales mediante las ventas obtenidas. Para ello se decidió trabajar en un modelo de desempeño ferial de las empresas expositoras que incluye la dimensión de ventas. El estudio es cuantitativo y el tipo de investigación es correlacional. Se utilizó el método hipotético deductivo y se determinó como instrumento de recolección de datos a la encuesta. Como resultado se observa que dentro de la dimensión de ventas existe una importante correlación positiva y significativa entre el nivel de ventas a clientes actuales y el nivel de ventas a nuevos clientes.

Palabras clave: Medición desempeño ferial; ferias internacionales; expositores.

\section{ABSTRACT}

International trade fairs are an international marketing instrument usually used by the exporting companies. Despite the benefits of international trade fairs, Peruvian exporting companies have a limited participation as exhibitors in them. There is no evidence to show how Peruvian exhibitors assessed their participation in international trade fairs. The main objective of the study is to analyze through which dimensions exhibiting companies evaluate their exhibition performance at international trade through sales results. Therefore, we decided to work with a model of trade fair performance of exhibiting companies including a dimension based on sales results. The study

\footnotetext{
Especialista en marketing internacional en Alemania. Investigador. Doctorando en la Universidad Técnica de Berlín, Alemania. Egresado de la Maestría en Administración de la UNMSM. Dirección: TU Berlin, Sekr. MAR 2-6, Marchstr. 23, 10587 Berlin, Alemania.
} 
is quantitative and the research is correlational. The hypothetical deductive method was used and survey was determined as data collection instrument. As a result it appears that within the dimension of sales there is a strong positive and significant correlation between the level of sales to existing customers and the level of sales to new customers.

Keywords: Trade fair performance; international trade fairs; exhibitors.

\section{INTRODUCCIÓN}

Las ferias internacionales han crecido en importancia como una estrategia viable de promoción y ventas (Smith et al., 2003). Por ejemplo, Harris (2000) reporta que solo en el año 1998, un millón y medio de empresas estadounidenses mercadearon sus productos a través de 4500 ferias internacionales para un número estimado de 102 millones de visitantes. Las ferias internacionales son generalmente usadas por las empresas exportadoras para promover sus productos a los mercados globales (Hansen, 2004; Power and Jansson, 2008). Kirchgeorg et al., (2010) definen a las ferias como "eventos que se llevan a cabo en días específicos, donde un número grande de empresas presentan sus productos y los venden a clientes ya existentes o potenciales".

La evidencia señala que la participación en ferias internacionales representa uno de los rubros más importantes dentro del presupuesto de marketing de negocios. En el caso de las empresas estadounidenses, la participación en ferias representa del $10 \%$ al $15 \%$ de sus presupuestos de marketing (Dekimpe et al., 1997, Smith et al. 2003). Más radical es el caso de las europeas, donde este rubro llega a más del $20 \%$ (Skallerud, 2010). Y el caso de las empresas asiáticas es similar, entendiendo que su presencia en los mercados internacionales es cada vez mayor.

Un estudio realizado por Hultsman (2001) indicaba que el desempeño ferial que los expositores esperaban tener se dividía en cuatro grandes categorías: los contactos, la información, el cierre y la visibilidad. Contacto implicaba tener la oportunidad de establecer contactos, conocer gente nueva, renovar viejos contactos y distribuir material. En Información, los expositores querían conocer nuevas ideas y nuevos productos. Cierre significaba para los expositores nuevos negocios y hacer las ventas. La última categoría estaba relacionada con ganar visibilidad. Algunos expositores consideraron que aunque no obtuvieron los resultados esperados, el hecho de que su empresa estaba presente en la feria ya era importante.

Las empresas expositoras de ferias internacionales no conocen las causas determinantes ni tampoco como medir los resultados conseguidos con su participación, a pesar que son imprescindibles para justificar los esfuerzos invertidos y para valorar la eficacia del desempeño ferial (Herbig et al., 1994).

En el caso peruano, no se conocen estudios previos sobre desempeño ferial de expositores. Por lo tanto ha sido la primera vez que se ha realizado una investigación sobre la evaluación del desempeño ferial de las empresas expositoras de las ferias internacionales de la Comisión de Promoción del Perú para la Exportación y el Turismo (PROMPERÚ) relacionándolo con la dimensión de ventas. Es posible analizar los casos específicos de aquellos que participan en ferias internacionales realizadas localmente.

En general, se puede notar la carencia de investigaciones orientadas a analizar el desempeño ferial, especialmente en el Perú, lo cual puede ser atribuido al hecho que los efectos inmediatos y de largo plazo de las ferias internacionales no son siempre medibles, y que muchas veces las empresas expositoras no realizan un seguimiento a los contactos empresariales que hubieran podido conseguir durante una feria internacional (Allen y Richman, 1992). Desde este punto de vista, puede decirse que en el ámbito ferial, la ausencia de estudios sobre el rendimiento de la inversión en eventos se puede entender por las siguientes razones (Mesonero y Garmendia, 2004):

- Muchas empresas ni siquiera fijan objetivos de participación.

- En aquellos casos en los que si se definen objetivos, estos suelen ser muy heterogéneos lo cual dificulta enormemente su medición.

- Las ferias se emplean en combinación con otras herramientas de marketing como el correo 
directo, la publicidad o la venta personal, lo cual complica enormemente el cálculo de su rentabilidad real dadas las interacciones resultantes.

- El ciclo de compra industrial es muy largo, por lo que la venta final no se sabe hasta qué punto se debe a la feria o a otros instrumentos de marketing.

Se plantea el siguiente problema de investigación:

¿Cómo influyen las "ventas" en la evaluación del desempeño ferial de las empresas expositoras peruanas en ferias internacionales?

La literatura señala que conseguir ventas es uno de los objetivos más importantes del desempeño de una empresa como expositor en una feria. Smith et al., (2004) confirmaron que la participación en una feria comercial puede acelerar la progresión de un cliente a través de la proceso de compra. Para el vendedor, esto se traduce en una realización más rápida del ciclo de ventas lo que mejora la eficiencia de la venta personal. Muchos pedidos se cierran en el propio stand. En concreto, se considera que, para cerrar una venta, la actividad ferial es muy eficiente, con una influencia solo por detrás de la recomendación de conocidos y la venta personal (Shipley et al., 1993). Además, el contacto lo inicia el propio cliente, no el vendedor, por lo que hay un interés manifiesto por parte del cliente. Muchos visitantes no son sólo compradores sino personas influyentes en la decisión de sus respectivas empresas, y estos son más difíciles de conocer y contactar (Dekimpe et al., 1997). La feria permite dar a conocer un nuevo producto en un espacio único a multitud de clientes potenciales de calidad (Jiménez et al., 2002). El lanzamiento sería mucho más costoso si tuviese que realizarse de forma independiente a la feria, ya que la inversión publicitaria necesaria para dar a conocer un nuevo producto es mucho mayor (Navarro, 2001).

Como respuesta a esta necesidad, el presente artículo tiene como objetivo analizar si las empresas expositoras determinan su desempeño en las ferias internacionales a través de las ventas obtenidas. Las empresas peruanas expositoras consideran su participación en ferias internacionales como un elemento importante de su plan de exportación y de marketing internacional. Por lo tanto, las ventas que pudieran conseguir las empresas peruanas expositoras a partir de su participación en ferias internacionales vendrían a ser relevantes a la hora de evaluar su desempeño ferial frente a la inversión realizada.

Se han encontrado pocos estudios previos sobre la medición del desempeño ferial. Una dificultad que existe radica en definir los indicadores apropiados de desempeño y su medición. Uno de ellos es el de Bonoma (1983) quien mencionó que existen dos clases de objetivos: objetivos de ventas y objetivos de no-ventas (por ejemplo, chequear el mercado para la aceptación de un producto, localizando potenciales distribuidores, fortalecer las relaciones con los clientes actuales y potenciales).

Kerin and Cron (1987) agruparon a las empresas por alto y bajo desempeño basados en objetivos de venta y de no-venta, examinado luego la extensión de la influencia del tipo de industria, la empresa y la estrategia utilizada que afectaban el desempeño ferial. Sólo cuatro de los trece predictores probaron ser estadísticamente significativos: número de productos, número de clientes, objetivos feriales escritos y el uso de ferias verticales. En este sentido, todos estos factores de estrategia ferial, señalaban la importancia de acciones gerenciales para producir resultados superiores.

\section{METODOLOGÍA}

Es una investigación aplicada no experimental y transversal. La medición de las personas involucradas se realizará una sola vez en el estudio, en un determinado momento. En el Perú, sólo hay tres ferias internacionales sectoriales que se organizan localmente, habiéndose considerado para el presente estudio dos de ellas correspondientes a los sectores textil-confecciones (Perú Moda) y artesanías (Perú Gift), los cuales son similares porque sus productos van a clientes finales.

La unidad de análisis es el empresario-expositor que ha participado en la feria internacional "Perú Moda" (sector textil y confecciones) o en la feria internacional "Perú Gift" (artesanías) de la edición 2010. Se ha elegido como corte para la aplicación del instrumento la edición 2010, pues permite contar con información más realista respecto a la permanencia a largo plazo de los expositores (para impedir una visión que se restrinja a los años más recientes).

La población está compuesta por empresas 
expositoras del sector textil-confecciones y de artesanías. Como se mencionó previamente, se han elegido estos sectores por estar relacionados con el mercado al que van orientadas dichas ferias. No se consideraron a los expositores de los subsectores joyería, calzado y que no sean productores o parte de la cadena de textil y confecciones en el caso de la feria internacional "Perú Moda"; de la misma forma no se consideraron a las asociaciones de artesanos ya que no es posible realizar una evaluación desempeño ferial al contar con productos de distintas empresas y artesanos individuales, en el caso de la feria internacional "Perú Gift".

Dado que se trabaja con toda la población, entonces se realizó un censo, comprendiendo 44 empresas las que fueron encuestadas. Estas empresas son el universo de los expositores que han venido participando de manera continua en ambas ferias internacionales desde el año 2008 al 2014 (en al menos 4 de las 7 ferias anuales celebradas).

Para el presente trabajo de investigación se recolectaron los datos mediante un cuestionario. El formato del cuestionario tiene preguntas cerradas, los ítems y preguntas se conforman considerando la escala de Likert de 5 niveles.

Para obtener la información proveniente de las encuestas aplicadas, se utilizaron los programas MS Excel 13 y SPSS 22 para el tratamiento y análisis descriptivo. Se utilizó el programa SPSS 22 para analizar los estadísticos de correlación y regresión simple y múltiple.

En primer lugar se ha trabajado con los métodos estadísticos multivariantes y el análisis multivariante.

- Como técnica exploratoria del MES se ha trabajado con el análisis factorial exploratorio (EFA) y el análisis de componentes principales (PCA).

- Como técnica confirmatoria se ha trabajado con el análisis factorial confirmatorio.

\section{RESULTADOS Y DISCUSIÓN}

El instrumento de recolección de datos finalmente desarrollado, contiene 2 variables ( 1 de ellas explicativa) y 6 indicadores en total, cada

Fig. 1. Modelo conceptual propuesto

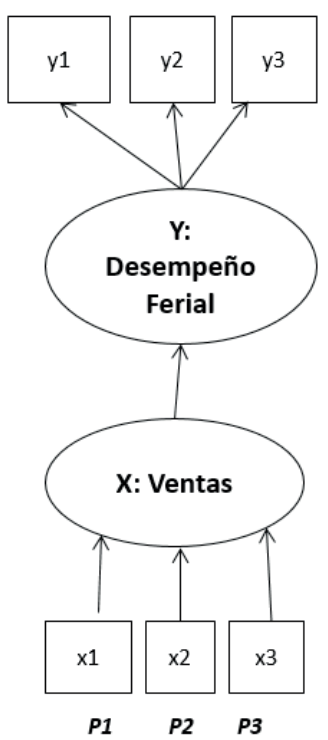

Leyenda:

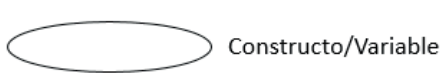

${ }_{P x x}$

Dimensión de la variable y pregunta del instrumento que la identifica

Fuente: Elaboración propia. 
uno de ellos relacionado a una pregunta o cuestionamiento presentado en dicho instrumento.

En el siguiente cuadro se presenta un resumen.

Cuadro 1. Variables y referencia a instrumento de recolección

\begin{tabular}{|c|c|c|c|}
\hline Variables & Definiciónconceptual & Indicadores & $\begin{array}{l}\text { Preguntasre- } \\
\text { lacionadasen } \\
\text { instrumento }\end{array}$ \\
\hline \multirow{3}{*}{$\begin{array}{l}\text { X1: } \\
\text { Ventas }\end{array}$} & \multirow{3}{*}{$\begin{array}{l}\text { Actividades relacio- } \\
\text { nadasconlasventas } \\
\text { tanto en el lugar de } \\
\text { la feria, así como } \\
\text { aquellas ventas } \\
\text { producidasdespués } \\
\text { de la feria pero re- } \\
\text { lacionadas con las } \\
\text { actividadesrealizadas } \\
\text { en la misma. }\end{array}$} & $\begin{array}{l}\text { Niveldeven- } \\
\text { tas a clientes } \\
\text { actuales }\end{array}$ & Preg. 01 \\
\hline & & $\begin{array}{l}\text { Niveldeven- } \\
\text { tas a nuevos } \\
\text { clientes }\end{array}$ & Preg. 02 \\
\hline & & $\begin{array}{l}\text { Resultado } \\
\text { de la intro- } \\
\text { ducción de } \\
\text { productos } \\
\text { nuevos du- } \\
\text { rante la feria }\end{array}$ & Preg. 03 \\
\hline \multirow{3}{*}{$\begin{array}{l}\text { Y: Des- } \\
\text { empeño } \\
\text { ferial }\end{array}$} & \multirow{3}{*}{$\begin{array}{l}\text { Desempeñoferialde } \\
\text { losexpositoresenlas } \\
\text { feriasinternacionales } \\
\text { PerúModayPerúGift } \\
\text { delaño2010,medido } \\
\text { a través del logro de } \\
\text { objetivosplaneadoso } \\
\text { cumplimientodeac- } \\
\text { tividadesplaneados } \\
\text { porlosexpositoresen } \\
\text { ambas ferias }\end{array}$} & $\begin{array}{l}\text { Valorización } \\
\text { del desem- } \\
\text { peñoferialde } \\
\text { la empresa } \\
\text { expositora } \\
\text { en la edición } \\
2010 \text { de la } \\
\text { feria }\end{array}$ & Preg. 04 \\
\hline & & $\begin{array}{l}\text { Satisfacción } \\
\text { del exposi- } \\
\text { tor con su } \\
\text { desempeño } \\
\text { comotalenla } \\
\text { feria }\end{array}$ & Preg. 05 \\
\hline & & $\begin{array}{l}\text { Inscripción } \\
\text { enlapróxima } \\
\text { edición de la } \\
\text { feria }\end{array}$ & Preg. 06 \\
\hline $\begin{array}{l}\text { Nombre } \\
\text { y cargo } \\
\text { delentre- } \\
\text { vistado }\end{array}$ & $\begin{array}{l}\text { Identificación del } \\
\text { entrevistado, para } \\
\text { validacióndeencues- } \\
\text { tayestablecimiento } \\
\text { de contacto con la } \\
\text { empresa }\end{array}$ & & Preg. 07 \\
\hline
\end{tabular}

Fuente: Elaboración propia

Con los resultados globales producto de la aplicación de 44 encuestas, se obtuvo las estadísticas descriptivas. Cabe señalar que no se ha perdido ningún resultado.

\section{Pruebas de fiabilidad al modelo Variables relacionadas a Ventas}

V.1: Nivel de ventas a clientes actuales

\section{V.2: Nivel de ventas a nuevos clientes}

V.3: Resultado de la introducción de productos nuevos durante la Feria

El Alfa de Cronbach es de 0.186 , mucho menor al 0.7 requerido para soportar la fiabilidad requerida. Se encuentran valores negativos debidos a una covarianza promedio negativa entre elemento, lo que viola los supuestos de fiabilidad del modelo.

La eliminación de la variable 3 incrementa el Alfa de Cronbach a 0.384 (desde los 0.186 actuales). Se deduce que existen problemas de codificación de las variables (la forma como se elaboraron las preguntas). Las variables no cumplen los supuestos de fiabilidad. Se evaluó la permanencia de las variables en el modelo.

\section{Análisis factorial confirmatorio del modelo planteado}

Las preguntas relacionadas a la Variable Ventas son:

V.1: Nivel de ventas a clientes actuales

V.2: Nivel de ventas a nuevos clientes

V.3: Resultado de la introducción de productos nuevos durante la Feria

Hay correlación positiva de 0.238 y significativa (6\%) entre V1 y V2.

Existe una importante correlación positiva y significativa entre el Nivel de ventas a clientes actuales y el Nivel de ventas a nuevos clientes (a pesar de que ambos son constructos distintos). No se podría explicar una relación entre ambas a partir de los constructos.

Hay una correlación positiva de 0.065 y poco significativa (33.7\%) entre V1 y V3.

Existe una correlación positiva poco significativa entre el Nivel de ventas a clientes actuales y el Resultado de la introducción de productos nuevos durante la feria (ambos son constructos distintos). Se puede entender que las ventas a clientes actuales se ven potenciadas por la introducción de nuevos productos durante la feria.

Hay una correlación negativa de -0.135 y poco significativa (19.2\%) entre V2 y V3.

Existe una correlación negativa poco significativa entre el Nivel de ventas a nuevos clientes y el Resultado de la introducción de productos nuevos durante la Feria (ambos son constructos 
distintos). Se puede interpretar que las ventas a nuevos clientes no se están generando a partir de la introducción de nuevos productos, sino que se basan principalmente en los productos ya reconocidos y tradicionales de la empresa.

Según el análisis de comunalidades, no debe extraerse ninguna variable. Según el análisis de matriz de componente, las variables V1 y V2 pertenecen a un mismo concepto.

La variable V3 pertenece a otro concepto distinto.

\section{Conclusiones referidas a las Variables Ventas:}

1. Las variables V1 y V2 pueden ser agrupadas en un solo componente (se utilizó la matriz de transformación de componente hallada).

2. La variable V3 pertenece a otro concepto, se tuvo que evaluar su significancia individual para saber si era relevante su permanencia en el modelo.

Con estas nuevas variables, se ha replanteado y ajustado el modelo, de tal forma que su nueva representación gráfica es la siguiente:
Las nuevas variables presentaron situaciones de normalidad, en el caso de Ventas 1, Ventas 2. Adicionalmente, para verificar que se mantiene una relación de dependencia entre las variables explicativas con la variable dependiente Desempeño, se desarrollaron correlaciones bivariadas.

Se evidenció claramente que la única variable que mostró correlación con el Desempeño, fue la variable Ventas 1 (0.344 al 0.05 de significancia, según Pearson; 0.309 al mismo de nivel de significancia según Kendall; y 0.367 al mismo nivel de significancia, según Spearman).

Cuadro 2. Resumen del modelo

\begin{tabular}{|c|c|c|c|c|}
\hline \multicolumn{5}{|c|}{ Resumen del modelob } \\
\hline Modelo & $\mathrm{R}$ & R cuadrado & $\begin{array}{l}\text { Rcuadrado } \\
\text { ajustado }\end{array}$ & $\begin{array}{c}\text { Error estándar } \\
\text { delaestimación }\end{array}$ \\
\hline 1 &, $364 a$ &, 132 &, 090 &, 53029 \\
\hline
\end{tabular}

a. Predictores: (Constante), Ventas2, Ventas 1

b. Variable dependiente: Desempeño

Fuente: elaboración propia

En resumen, el modelo replanteado con todas sus variables presentó un $\mathrm{R}^{2}$ de 0.132 y un $\mathrm{R}^{2}$ ajustado de 0.09. Se realizó el Análisis de la Varianza (ANOVA, por sus siglas en inglés), que arrojó

Figura 2. Modelo final ajustado.

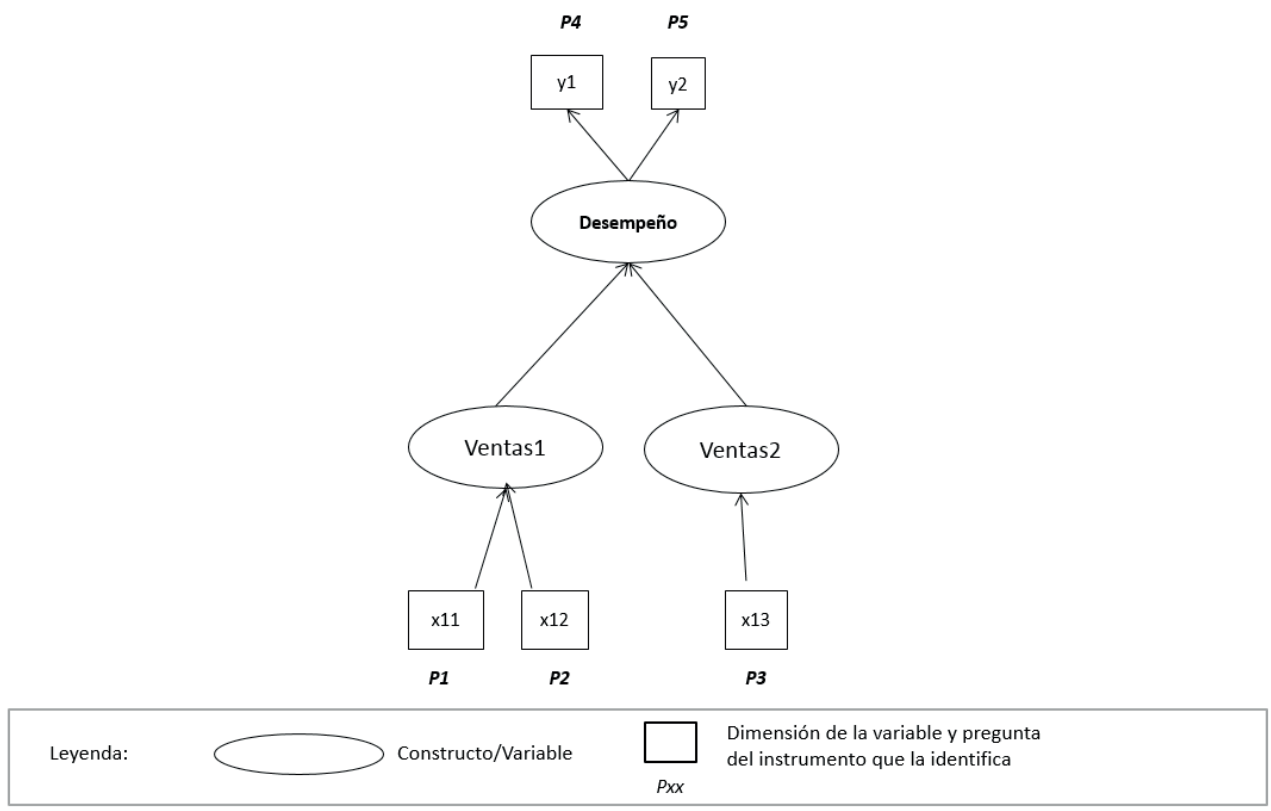

Fuente: elaboración propia 
como resultado un valor $\mathrm{F}$ de 3.129, teniendo un valor aceptable para una regresión múltiple con 2 variables explicativas y 44 valores.

Cuadro 3. Análisis de la Varianza ${ }^{\mathrm{a}}$

\begin{tabular}{|c|c|c|c|c|c|c|}
\hline & Modelo & $\begin{array}{l}\text { Suma de } \\
\text { cuadrados }\end{array}$ & $\mathrm{Gl}$ & $\begin{array}{c}\text { Media } \\
\text { cuadrática }\end{array}$ & $F$ & Sig. \\
\hline \multirow{3}{*}{1} & Regresión & 1,760 & 2 & 880 & 3,129 &, $054 b$ \\
\hline & Residuo & 11,530 & 41 & 281 & & \\
\hline & Total & 13,290 & 43 & & & \\
\hline
\end{tabular}

a. Variable dependiente: Desempeño

b. Predictores: (Constante), Ventas2, Ventas 1

Fuente: Elaboración propia.

En tal sentido, se puede señalar de manera contundente que la única variable con cierto nivel de significancia es Ventas1. Ventas2 no demuestra ser explicativa del desempeño ferial.
Resultados prueba de hipótesis específica a las regresiones del modelo ajustado

El valor de la " $t$ " de Student para Ventas 1 es de 2.323 , con una significancia a nivel de $97.5 \%$. Por tanto, el nivel de ventas a clientes actuales y el nivel de ventas a nuevos clientes se relacionan directamente con el desempeño ferial de las empresas expositoras peruanas durante su participación en las ferias internacionales Perú Moda y Perú Gift del año 2010. El nivel de ventas a clientes actuales y el nivel de ventas a nuevos clientes influyen en la valorización de la empresa expositora de su participación y también influye en la satisfacción del expositor con su desempeño en la feria.

El valor de la "t" de Student para Ventas 2 es de -0.819 , pero no tiene una significancia relevante. Por tanto, el resultado de la introduc-

Cuadro 4. Coeficientes ${ }^{\mathrm{a}}$

\begin{tabular}{|c|c|c|c|c|c|c|c|c|}
\hline & \multirow[t]{2}{*}{ Modelo B } & \multicolumn{2}{|c|}{ Coeficientesnoestandarizados } & \multirow[t]{2}{*}{ Coeficientesestandarizados } & \multirow[t]{2}{*}{$t$} & \multirow{2}{*}{$\begin{array}{c}\text { Sig. } \\
\text { Límiteinferior }\end{array}$} & \multicolumn{2}{|c|}{$\begin{array}{c}\text { 95.0\%intervalodeconfianza } \\
\text { para B }\end{array}$} \\
\hline & & Error estándar & Beta & & & & Límitesup & \\
\hline \multirow{3}{*}{1} & (Constante) & 3,163 & ,793 & & 3,987 &, 000 & 1,561 & 4,766 \\
\hline & Ventas1 &, 336 &, 145 & ,338 & 2,323 &, 025 &, 044 &, 628 \\
\hline & Ventas2 &,- 123 &, 151 &,- 119 &,- 819 & 418 &,- 427 & 181 \\
\hline
\end{tabular}

a. Variable dependiente: Desempeño

Fuente: Elaboración propia

El modelo ajustado resultante es:

Desempeño $=3.163+0.336$ Ventas $1-0.123$ Ventas 2

\section{Pruebas de hipótesis}

Se realizaron las pruebas de hipótesis a las regresiones del modelo ajustado, cuyo resumen se presenta a continuación:

Figura 3. Resumen de resultados del modelo ajustado

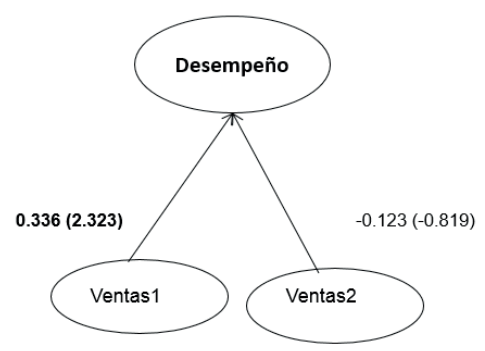

Fuente: Elaboración propia. ción de productos nuevos durante la feria no se relaciona directamente con el desempeño ferial de las empresas expositoras peruanas durante su participación en las ferias internacionales Perú Moda y Perú Gift del año 2010. La introducción de productos nuevos durante la feria no influye en la valorización de la empresa expositora de su participación y ni tampoco influye en la satisfacción del expositor con su desempeño en la feria.

\section{Presentación de resultados y discusión Resultados orientados al objetivo planteado}

Objetivo planteado: Determinar cómo se relacionan las "ventas" y el desempeño ferial de las empresas expositoras peruanas en las ferias internacionales

Según el resultado se observa que:

El nivel de ventas a clientes actuales y el nivel de ventas a nuevos clientes influyeron en la valorización de la empresa expositora de su participación y también influyeron en la satisfacción del expositor con su desempeño en la feria. 
Este resultado no debe sorprender, dado que "conseguir ventas" es uno de los objetivos más importantes de una feria. Kim (2005), rescató algunos resultados previos donde se reportaba que realizar ventas era una de las principales categorías de lo que las empresas expositoras deseaban ganar en una feria internacional. La categoría "realizar ventas" incluía conseguir nuevos negocios (Ventas2 para esta investigación) y hacer ventas (Ventas1 en nuestro caso).

El peso del factor ventas en la participación ferial se evidencia no solo en Perú, sino también en otros países latinoamericanos. Un estudio reciente de la Universidad de Chile (Olivares, 2014) midió la percepción sobre las ferias internacionales patrocinadas por ProChile según Rubro de Empresas Participantes, alcanzando el ítem "realizar ventas" una media de 71 puntos sobre un máximo de 100.

La no contundencia de los resultados del presente estudio en el Perú (nivel de significancia no llega al 1\%), ha sido evidenciada previamente en otros estudios, aunque lo real es que no hay literatura previa que demuestre fehacientemente la eficacia de una feria como herramienta de actividad de marketing para generar ventas (Keswell, 2010).

Blythe (2010) considera que la no fiabilidad de los resultados se debe en gran parte al hecho de que pocos visitantes a las ferias tienen un papel relevante en el proceso de compra, e incluso los que lo hacen no se encuentran participando en el ciclo de compra, por lo cual no están en la capacidad de hacer cualquier compromiso in situ. Los expositores feriales con frecuencia actúan como si no fueran conscientes de que la mayoría los visitantes no son los compradores. Como resultado, algunos expositores dejan la feria sin haber logrado sus objetivos, y muchos visitantes se vienen quejando sobre el "enfoque de los expositores en demasía hacia las ventas".

La introducción de productos nuevos durante la feria no influyó en la valorización de la empresa expositora de su participación y tampoco influyó en la satisfacción del expositor con su desempeño en la feria.

Tanner y Chonko (1995) ya habían resaltado que el objetivo principal de los expositores de ferias eran las ventas (alrededor del 62\%), muy por encima de la introducción de nuevos productos o generación de nuevos negocios.

\section{Limitaciones del estudio}

1. Una limitación del presente estudio es que se observa que pocas empresas exportadoras o con potencial de exportación que participan regularmente en las ferias internacionales organizadas localmente. Esto, unido al hecho de que en el Perú se desarrollan pocas ferias internacionales organizadas localmente, lo que hace menos factible contar con un campo amplio donde desarrollar este tipo de investigaciones con carácter cuantitativo.

2. Metodológicamente se han cumplido parcialmente algunos supuestos requeridos para la aplicación del análisis factorial confirmatorio del modelo presentado.

\section{CONCLUSIONES}

1. Las empresas expositoras peruanas efectivamente evalúan su desempeño ferial en ferias internacionales organizadas por PROMPERÚ en el año 2010 a través de las ventas realizadas a partir de ambas ferias.

2. Las empresas expositoras peruanas evalúan su desempeño ferial principalmente a través del nivel de ventas a clientes actuales y a nuevos clientes, los cuales influyen en la valorización de la empresa expositora sobre su participación y también en la satisfacción de la misma con su desempeño en la feria.

3. La introducción de productos nuevos durante la feria no influye en la valorización de la empresa expositora de su participación y ni tampoco influye en la satisfacción de la misma con su desempeño en la feria. Estudios previos ya habían resaltado que el objetivo principal de los expositores de ferias eran las ventas, muy por encima de la introducción de nuevos productos o generación de nuevos negocios.

\section{REFERENCIAS BIBLIOGRÁFICAS}

Allen, S. y Richman, T. (1992). Stretching the tradeshow budget. Inc., 01628968, Vol. 14, 5

Bonoma, T. V. (1983). Get More Out Of Your Trade Show. Harvard Business Review. (61), pp. 75-83.

Blythe, J. (2010). Trade fairs as communication: a new model. Journal of Business \& Industrial Marketing, 25/1, pp. 57-62. 
Dekimpe, M. G.; François, P.; Goplakrishna, S.; Lilien, G. L. y Van Den Bulte, C. (1997). Generalizing About Trade Show Effectiveness: A Cross-National Comparison. Journal Marketing, (61), pp. 55-64.

Herbig, P., O’Hara, B. y Palumbo, F. (1994). Measuring Trade Show Effectiveness: An Effective Exercise?, Industrial Marketing Management, vol. 23, pp. 165-70.

Hansen, K. (2004). Measuring performance at trade shows. Scale development and validation, Journal of Business Research, (57), pp. 1-13.

Harris, D. (2000). The shows will go on, Journal Sales \& Marketing Management, vol. 152, $\mathrm{N}^{\circ}$ 5, pp. 85-88.

Hultsman, W. (2001). From the eyes of an exhibitor: Characteristics that make exhibitions a success for all stakeholders. Journal of Convention \& Exhibition Management, 3(3), pp. 27-44.

Jiménez, J. F.; Cazorla, I. M. y Linares, E. (2002). Ferias Comerciales en España. Un análisis sectorial, Revista Distribución y Consumo, 61, enero-febrero, pp. 61-71.

Kerin, R.A. y Cron, W.L. (1987). Assessing trade show functions and performance: an exploratory study. Journal of Marketing, Vol. 51, pp. 87-94.

Keswell, D. (2010). The effectiveness of trade show marketing capabilities on the financial performance of South African companies. South Africa: University of Pretoria.

Kim, Y. K. (2005). Korean Exhibitors' Behavior in Overseas Exhibitions, Working Paper, University of Nevada, Las Vegas.

Kirchgeorg, M.; Springer, C. y Kastner, E. (2010). Objectives for successfully participating in trade shows. Journal of Business \& Industrial Marketing, 25 (1), pp. 63-72.
Mesonero, M. y Garmendia, F. (2004). Comunicaciones integradas feriales o cómo planificar con éxito una feria industrial, Revista de Dirección y Administración de Empresas, 11, pp. 109-129.

Navarro, F. (2001). Estrategias de marketing ferial. Madrid: ESIC.

Olivares, P. (2014). Las Ferias Internacionales Patrocinadas por ProChile: ¿Cuál es la Percepción de las Empresas Participantes? Seminario de estudio de caso para optar al grado de Magíster en Estrategia Internacional y Política Comercial. Instituto de Estudios Internacionales Universidad de Chile. Junio 2014.

Power, D. y Jansson, J. (2008). Cyclical Clusters in global Circuits: Overlapping Spaces in Furniture Trade Fairs. Economic Geography, 84 (4), pp. 423-448.

Shipley, D.; Egan C. y Wong, K. S. (1993), Exhibiting Strategy and Implementation. International Journal of Advertising, 12, pp. 117-130.

Skallerud, K. (2010). Structure, Strategy and Performance of Exhibitors at Individual Booths versus Joint Booths. Journal of Business $\mathcal{E}$ Industrial Marketing, 25(4), pp. 259-67.

Smith, T. M.; Hama, K., y Smith, P. M. (2003). The effect of successful trade show attendance on future show interest: exploring Japanese attendee perspectives of domestic and offshore international events. Journal of Business \& Industrial Marketing, 18(4/5), pp. 403-18.

Smith, T. M.; Gopalakrishna, S., y Smith, P. M. (2004). The complementary effect of trade shows on personal selling. International Journal of Research in Marketing 21, pp.61-76.

Tanner, J. y Chonko, L. (1995). Trade Show Objectives, Management, and Staffing Practices. Industrial Marketing Management, vol. 24, pp. 257-264. 